\title{
"Del miedo a la resiliencia". Estudio fenomenológico sobre el impacto de la pandemia por COVID-19 en cuidadoras de pacientes dependientes en hemodiálisis
}

\author{
Ana Casaux-Huertas ${ }^{1,2}$, Antonio Ochando-García ${ }^{3}$, Enric Limón-Cáceres ${ }^{4,5}$, Dolores Andreu-Périz ${ }^{4}$ \\ ${ }^{1}$ Escuela de Enfermería Fundación Jiménez Díaz. Campus de Villalba. Madrid. España \\ ${ }^{2}$ Fundación Íñigo Álvarez de Toledo. Madrid. España \\ ${ }^{3}$ Hospital Universitario Fundación Alcorcón. Madrid. España \\ ${ }^{4}$ Universitat de Barcelona. España \\ ${ }^{5}$ Programa VINCat (Control de las Infecciones nosocomiales en Cataluña)
}

Como citar este artículo: Casaux-Huertas A, Ochando-García A, Limón-Caceres E, Andreu-Periz D. "Del miedo a la resiliencia". Estudio fenomenológico sobre el impacto de la pandemia de COVID-19 en cuidadoras de pacientes dependientes en hemodiálisis.

Enferm Nefrol. 2021 Jul-Sep;24(3):250-60

\section{Resumen}

Objetivo: profundizar en el conocimiento sobre las vivencias y el soporte percibido en cuidadoras primarias que atendieron a pacientes dependientes en tratamiento con hemodiálisis durante el periodo de pandemia por COVID-19 en la ciudad de Madrid.

Material y Método: estudio transversal efectuado con diez cuidadoras primarias de pacientes en tratamiento de hemodiálisis que habían proporcionado cuidados durante el periodo de marzo 2020 a junio 2021. La recolección de los datos se realizó mediante entrevistas semiestructuradas a distancia hasta conseguir la saturación de las unidades de significado. Se realizó un análisis cualitativo fenomenológico mediante el método de siete pasos de Colaizzi.

Resultados: del análisis de los discursos emergieron dimensiones asociadas a diferentes subcategorías: miedo inicial sobre la enfermedad, pérdida de libertad, riesgo percibido durante el transporte, soporte percibido por parte de los centros de diálisis, capacidad de resiliencia, miedo a la muerte e impacto de la vacunación.

\section{Correspondencia:}

Antonio Ochando García

E-mail: aochandoseden@gmail.com
Conclusiones: en la etapa álgida del brote epidémico las vivencias de las cuidadoras estuvieron muy influidas por el desconocimiento de la enfermedad y el miedo al contagio. Adaptaron medidas de auto prevención para mantener a salvo a su familiar. Apreciaron la seguridad proporcionada por los profesionales de los centros. Les preocupaba los riesgos inherentes a los traslados y la falta de soporte de los servicios sociales. Son conscientes de su propia fragilidad y la de la persona cuidada y no perciben grandes cambios ni en su forma de vida ni en su esperanza de futuro, pese a la vacunación y otros adelantos científicos.

PALABRAS CLAVE: cuidadores; COVID-19; pandemia; hemodiálisis; experiencias de vida.

\section{"From fear to resilience". Phenomenological study on the impact of the COVID-19 pandemic on caregivers of dependent hemodialysis patients}

\section{Abstract}

Objective: Deepening knowledge of the experiences and support perceived in primary caregivers who attended patients in hemodialysis treatment during the pandemic period for COVID-19 in the city of Madrid. 
Material and Method: Cross-sectional study conducted with ten primary caregivers of patients in hemodialysis treatment who had provided care during the period from March 2020 to June 2021. The collection of the data was carried out through semi-structured distance interviews to achieve saturation of the units of meaning. A phenomenological qualitative analysis was carried out using Colaizzi's seven-pass method.

Results: From the analysis of the speeches, dimensions associated with different subcategories emerged: initial fear about the disease, loss of freedom, perceived risk during transport, perceived support from dialysis centres, resilience, fear of death and impact of vaccination.

Conclusions: At the height of the outbreak, caregivers' experiences were strongly influenced by their lack of knowledge of the disease and fear of infection. They adapted self-prevention measures to keep their family member safe. They appreciated the safety provided by the professionals in the centres. They were concerned about the risks inherent in transfers and the lack of support from social services. They are aware of their own and the cared-for person's frailty and do not perceive major changes in their way of life or in their hope for the future, despite vaccination and other scientific advances.

KEYWORDS: caregivers; COVID-19; pandemic; hemodialysis; life experiences.

\section{Introducción}

Al inicio de 2020 el mundo se vio inmerso en una situación que, aunque descrita en la historia de la civilización, sus características se veían impensables en una sociedad avanzada del siglo XXI ${ }^{1}$. Palabras como confinamiento o cuarentena solo se contemplaban en el ambiente sanitario de forma puntual y circunscrita al control de ciertas infecciones ${ }^{2}$. La pandemia de COVID-19 ha tenido un impacto muy importante en España, siendo uno de los países significativamente más afectados en cuanto a contagios y letalidad ${ }^{3}$.

Entre los grupos más vulnerables se encuentran los pacientes en diálisis debido a su inmunidad deprimida, la patología cardiovascular asociada, así como a su necesidad de recibir tratamiento de forma periódica en centros de diálisis ${ }^{4}$. A este riesgo se le debe sumar la necesidad de acudir con regularidad en transporte colectivo a un centro sanitario, en el que comparten espacios durante varias horas con otros pacientes y son atendidos por personal sanitari $0^{5,6}$, es por ello que los centros y las unidades de diálisis han requerido de protocolos específicos de prevención al considerarse zonas de alto riesgo de transmisión ${ }^{7,8}$. La atención a estos pacientes ha hecho necesario reconciliar los protocolos de control de la infección con las necesidades intrínsecas del tratamiento?

La gestión de la pandemia, durante las sucesivas olas pandémicas, se ha centrado en evitar la transmisión nosocomial y comunitaria ${ }^{3}$. Se han concentrado los esfuerzos en evitar las hospitalizaciones, los ingresos en cuidados intensivos y reducir la mortalidad atribuible ${ }^{7}$. La pandemia sin embargo ha tenido repercusiones en muchos ámbitos de la salud y uno de los efectos más importantes ha sido la afectación de la salud mental de la población y específicamente la de aquellos pacientes y cuidadores de grupos de riesgo que tenían una mayor vulnerabilidad ${ }^{5}$.

En informes recientes se manifiesta que en España, debido a la COVID-19 un porcentaje superior al 80 por ciento de los cuidadores familiares de personas con algún grado de dependencia afirma que su labor durante la pandemia les ha causado "más desgaste que nunca", y el 70 por ciento asegura que su salud mental y emocional se han deteriorado durante este tiempo, siendo estos porcentajes superiores a los de otros países del entorno ${ }^{10-12}$. Durante este periodo ha habido iniciativas por parte de diferentes actores del sistema de salud para dar soporte a los cuidadores, pero estas iniciativas han sido limitadas y las medidas de soporte que ya estaban instauradas se han visto afectadas por la situación de restricciones y medidas de prevención que se han activado a nivel social (ayuda de cuidadoras profesionales, centros de día, apoyo económico, etc.... $)^{7}$. Entre estas estrategias descritas para dar apoyo al cuidador destacan la educación psicoeducativa, las aportaciones económicas o la ayuda de un cuidador profesional ${ }^{9}$.

Las personas que cuidan en el hogar a los pacientes en tratamiento de hemodiálisis perciben y expresan una sobrecarga que está relacionada con la evolución del paciente, y que se acrecienta a medida que aumenta su nivel de dependencia ${ }^{10}$. El riesgo de contagio de SARS-CoV-2, debido a la alta prevalencia que se dio en las unidades de diálisis desde los primeros meses de la pandemia, ha supuesto que los pacientes y sus familiares hayan percibido las sesiones de diálisis como una situación de riesgo alto de contraer la enfer- 
medad $^{13}$. Las necesidades de estos pacientes y de sus cuidadores, ampliamente estudiadas en la literatura científica, han sido modificadas por la implantación de medidas preventivas adicionales ${ }^{11}$.

Los servicios asistenciales y las sociedades científicas relacionadas con el paciente renal han elaborado protocolos y recomendaciones específicas para evitar el contagio y promover un entorno seguro en el hogar; no obstante, en estas nuevas circunstancias las vivencias de sus cuidadores, figura clave en su atención, deben ser analizadas para fomentar su resiliencia y capacidad de actuación.

\section{Objetivo}

Profundizar en el conocimiento sobre las vivencias y el soporte percibido en cuidadoras primarias que atendieron a pacientes dependientes, en tratamiento con hemodiálisis, durante el periodo de mayor prevalencia de la pandemia por COVID-19 en la ciudad de Madrid (marzo 2020-junio 2021).

\section{Participantes y Método}

\section{Diseño}

Se desarrolló un estudio exploratorio transversal mediante análisis de contenido cualitativo, utilizando el método fenomenológico de Colazzi.

\section{Ámbito y sujetos}

Se pretendía estudiar el fenómeno en una zona específica de Madrid, dada la situación epidemiológica de alta incidencia que se vivió durante el periodo en estudio, de enero 2020 a junio 2021. El tamaño de la muestra se determinó de forma progresiva durante el transcurso de la investigación, hasta alcanzar la saturación de la información. Como criterios de inclusión se tuvieron en cuenta que fueran cuidadoras/es primarias de personas dependientes no institucionalizadas, que estuvieran en tratamiento con hemodiálisis (HD) en uno de los dos centros de referencia del estudio y que dieran su consentimiento a participar de forma voluntaria en la investigación. Se excluyeron aquellas personas que presentaban problemas de comunicación, en las que no era posible mantener la entrevista.

Periodo de estudio: las entrevistas tuvieron lugar entre el 10 y el 30 de julio de 2021. La información recogida hacía mención al periodo de marzo 2020 a junio 2021.

\section{Método de recogida de información}

La recogida de información se llevó a cabo mediante entrevistas individuales semiestructuradas. Se construyó un formato de entrevista que fue sometido al juicio de tres expertos en enfermería nefrológica. Los contenidos de los ejes de indagación de dicha entrevista se elaboraron a partir de la literatura revisada y de la opinión de expertos (tabla 1). Se establecieron 6 ejes: servicios de salud, medidas de prevención, miedo a la muerte, conocimiento sobre la enfermedad, emociones y expectativas.

\section{Procedimiento}

Ante la situación de riesgo de transmisión de la enfermedad, las entrevistas se realizaron mediante llamada telefónica. Se llevaron a cabo un total de 10 entrevistas, con una duración que osciló entre 14 y 46 minutos con las que se llegó a la saturación de los datos. EI perfil de las cuidadoras se detalla en la tabla 1. En la tabla 2 se describen las principales características de los pacientes en HD.

\section{Análisis de los datos}

Tras la transcripción literal de las entrevistas se realizó el análisis de contenido utilizando el método 52. Se utilizó como método de codificación informática el programa NUD-IST. Se adjudicó a los participantes un código alfanumérico $(\mathrm{Cl}, \mathrm{C} 2, \mathrm{C} 3, \mathrm{C} 4 \ldots)$ que sirvió para el registro de los datos, para elaborar categorías y para referenciar frases que se extrajeron durante las entrevistas.

\section{Aspectos éticos:}

El estudio fue revisado y aprobado por el Comité de Ética e Investigación del Hospital Universitario Fundación Alcorcón. Una vez elegida la muestra de estudio, los propios investigadores se encargaron de informar a los seleccionados sobre la investigación, pedirles el consentimiento informado de forma verbal, así como indicarles la confidencialidad del estudio, además de la libertad de poder abandonarlo en cualquier momento. Se mantuvo el anonimato de los sujetos utilizando en todo momento un código alfanumérico. Las grabaciones se trasladaron inmediatamente a los ordenadores personales de los investigadores, y una vez transcritas fueron eliminadas.

\section{Criterios de rigor:}

El rigor del estudio se ha basado en los criterios que definen Lincoln y Guba ${ }^{14}$. El diseño del estudio, el material transcrito analizado, acompañado de las notas de campo, ha permitido la triangulación por otros investigadores para garantizar la credibilidad. La transferibilidad del estudio vino dada por la descripción del fenómeno y las 
Tabla 1. Perfil de los participantes en las entrevistas.

\begin{tabular}{|c|c|c|c|c|c|c|c|c|}
\hline Codificación & Edad & Sexo & Estado civil & Nivel de estudios & $\begin{array}{l}\text { Grado de } \\
\text { parentesco/ } \\
\text { relación con } \\
\text { el paciente }\end{array}$ & $\begin{array}{l}\text { Tiempo } \\
\text { desde que } \\
\text { comenzó } \\
\text { a cuidar } \\
\text { al paciente }\end{array}$ & $\begin{array}{l}\text { ¿Vive con } \\
\text { el paciente? }\end{array}$ & $\begin{array}{l}\text { ¿En algún } \\
\text { momento ha } \\
\text { sido positivo } \\
\text { COVID-19 } \\
\text { confirmado? }\end{array}$ \\
\hline $\mathrm{Cl}$ & 62 & Mujer & Casada & Bachillerato & Sobrina & 3 años & Sí & Sí \\
\hline $\mathrm{C} 2$ & 70 & Mujer & Casada & Primaria & Esposa & 2 años & Sí & No \\
\hline $\mathrm{C} 3$ & 53 & Mujer & Soltera & Formación profesional & $\mathrm{Hija}$ & 20 años & Sí & No \\
\hline C4 & 76 & Mujer & Casada & Primaria & Esposa & 2 años & Sí & No \\
\hline C5 & 77 & Mujer & Soltera & Grado Medio & Pareja & 7 meses & Sí & No \\
\hline C6 & 71 & Mujer & Casada & Bachillerato & Esposa & 19 años & Sí & No \\
\hline $\mathrm{C7}$ & 75 & Mujer & Casada & Bachillerato & Esposa & 8 años & Sí & Sí \\
\hline C8 & 89 & Hombre & Casado & Leer y escribir & Esposo & 23 años & Sí & No \\
\hline $\mathrm{co}$ & 68 & Mujer & Casado & Bachillerato & Esposa & 5 años & $\mathrm{Si}$ & No \\
\hline $\mathrm{ClO}$ & 52 & Mujer & Casada & Bachillerato & Hija & 11 años & No & No \\
\hline
\end{tabular}

Tabla 2. Características de los pacientes en hemodiálisis.

\begin{tabular}{|c|c|c|c|c|c|c|}
\hline Codificación & Edad paciente & Sexo paciente & Fecha inicio HD & Barthel & Estado civil \\
\hline C1 & 88 & Mujer & $13 / 11 / 2017$ & 50 & Viuda \\
\hline C2 & 71 & Hombre & $27 / 12 / 2002$ & 45 & 35 & Casado \\
\hline C3 & 90 & Hombre & $28 / 12 / 2002$ & 40 & Casado \\
\hline C4 & 74 & Hombre & $15 / 04 / 2019$ & 70 & Soltero \\
\hline C5 & 63 & Hombre & $12 / 12 / 2018$ & 40 & Casado \\
\hline C6 & 72 & Hombre & $28 / 11 / 2019$ & 50 & Casado \\
\hline C7 & 75 & Hombre & $26 / 04 / 2017$ & 20 & Casado \\
\hline C8 & 88 & Mujer & $25 / 06 / 1999$ & 5 & Casado \\
\hline C9 & 67 & Hombre & $29 / 07 / 2013$ & $06 / 04 / 2016$ & 20 & Casado \\
\hline C10 & 88 & Hombre & & & 5 \\
\hline
\end{tabular}

características de la muestra. Por otra parte, la reflexividad provino de la recogida y el análisis de los datos, garantizando su estabilidad en caso de repetir la misma investigación. Por último, se ha tenido presente el concepto de confirmabilidad, al referenciar nuestros resultados con otros investigadores que han obtenido resultados similares.

\section{Resultados}

Se entrevistaron 9 cuidadoras y 1 cuidador. El tiempo medio de la entrevista fue de $22,10 \pm 9,90$ minutos (rango entre 14-46). La edad media era de 69,35 $\pm 11,29$ años (rango entre 52-89). La media de años ejerciendo como cuidadoras era de 9,30 $\pm 8,44$ años (rango entre 7 meses y 23 años). Las 10 cuidadoras/es vivían con el paciente, de las cuales únicamente dos declararon haber sido diagnosticadas de COVID-19. Estas mismas personas expusieron que sus familiares en diálisis también habían dado positivo en la enfermedad. Cinco cuidadoras verbalizaron tener alteraciones del estado emocional (50\%).

El análisis del contenido de los datos obtenidos condujo a la identificación de 5 grupos temáticos y 17 subtemas (tabla 3). 
Tabla 3. Características de los pacientes en hemodiálisis.

\begin{tabular}{|l|l|}
\hline Grupos temáticos & Subtemas \\
\hline $\begin{array}{l}\text { Servicios de salud recibidos } \\
\text { durante este periodo }\end{array}$ & $\begin{array}{l}\text { Servicios recibidos } \\
\text { Respuesta de los centros de diálisis } \\
\text { Transporte sanitario }\end{array}$ \\
\hline $\begin{array}{l}\text { Impacto de las medidas } \\
\text { preventivas }\end{array}$ & $\begin{array}{l}\text { Limitación de la libertad } \\
\text { Introducción de las medidas preventivas } \\
\text { Aislamiento social y convivencia }\end{array}$ \\
\hline Mortalidad & $\begin{array}{l}\text { Percepción de riesgo de morir, } \\
\text { del propio cuidador y del familiar } \\
\text { Muerte de personas próximas }\end{array}$ \\
\hline Enfermedad por CoVID-19 & $\begin{array}{l}\text { Riesgo de transmisión } \\
\text { Desconocimiento } \\
\text { Exacunación }\end{array}$ \\
$\begin{array}{l}\text { Experiencia personal } \\
\text { Confianza en la vacuna } \\
\text { Situación post vacunación }\end{array}$ \\
\hline Emociones y expectativas & $\begin{array}{l}\text { Grado de miedo y ansiedad } \\
\text { Aumento de hábitos no saludables } \\
\text { Alteración del sueño } \\
\text { Proyecciones futuras }\end{array}$ \\
\hline
\end{tabular}

\section{Tema: servicios de salud recibidos durante este periodo}

Dentro de esta categoría se han incluido las referencias a los servicios recibidos. Se ha recogido de forma explícita en la entrevista la evolución percibida por parte de los cuidadores desde las etapas iniciales hasta el periodo post vacunación.

\section{Subtema: servicios recibidos}

Las cuidadoras del estudio experimentaron al inicio de la pandemia una ausencia de ciertos servicios, y a su vez una pérdida de control sobre la gestión que se estaba realizando de su familiar (C3-C8). El desconcierto sobre cómo actuar, cuando no podían seguir llevando las rutinas habituales con sus familiares, generó ansiedad y miedo a que no fueran bien cuidados. El "miedo ante lo desconocido" estuvo presente durante las primeras fases de la pandemia, aunque posteriormente ese miedo fue disminuyendo hasta quedar integrado en una nueva rutina $(\mathrm{C} 2, \mathrm{C6})$. Una de sus principales preocupaciones fue la pérdida de ciertos servicios, que afectaban directamente a su rutina diaria, y no poder disponer de servicios de urgencias si la situación de su familiar se deterioraba (C3, C8). La necesidad de solicitar el soporte de los servicios sociales ante la ausencia de sus propias redes familiares, ha supuesto un gran desgaste debido principalmente a que estas ayudas han sido mínimas o inexistentes $(\mathrm{Cl}, \mathrm{C7}, \mathrm{C} 9)$. Esta situación motivó sentimientos de desamparo y de redefinición de los recursos disponibles.

\section{Subtema: respuestas de los centros de diálisis}

Las cuidadoras han percibido que los servicios han funcionado con regularidad y eso les ha transmitido seguridad durante todo este tiempo. Los cambios en las dinámicas asistenciales que fueron necesarios en los primeros periodos han sido integrados en sus rutinas y en las de sus familiares. Las participantes consideran necesario expresar su agradecimiento a los equipos humanos en numerosas ocasiones, porque son conscientes que han tenido que trabajar en situaciones excepcionales (C2-C5-C8). Tienen muy presente que el tratamiento que reciben sus familiares es necesario realizarlo en este tipo de centros y no se planteaban alternativas, aunque hubiera un riesgo importante de contraer la enfermedad $(\mathrm{C} 1, \mathrm{C} 2, \mathrm{C} 3, \mathrm{C} 4$, $\mathrm{C} 5, \mathrm{C} 9$ ). Hay un aspecto que destacan cuando hablan de los servicios, y es la necesidad de dotarlos de más personal (C1). Este aumento del ratio lo expresan más aquellas cuidadoras que tienen a sus familiares realizando la diálisis en una unidad hospitalaria (C8-C9-C10). Un aspecto que también verbalizan es el impacto que les ha provocado la desaparición de la posibilidad de realizar diálisis durante breves periodos en otros centros, durante los periodos vacacionales, lo que ha limitado mucho su posibilidad de disponer de unos días de cambio en las rutinas (C1-C4).

\section{Subtema: transporte sanitario}

El riesgo percibido durante el transporte sanitario ha sido un elemento común en la mayoría de las cuidadoras entrevistadas. Este riesgo, al tener que utilizar transportes compartidos, ha motivado incluso que dos de las familias entrevistadas optaran por realizar el transporte en taxi privado (C4-C7). La valoración sobre el transporte difiere mucho de la realizada sobre los servicios de diálisis, y es en general muy negativa. El hecho de no poder acompañar a sus familiares, la ausencia de información sobre las rutas y los horarios, han sido tres de los aspectos que con mayor unanimidad habían provocado una sensación de pérdida de control y aumento del riesgo $(\mathrm{Cl}, \mathrm{C} 2, \mathrm{C} 5$, C7, C8). Si bien la adaptación se ha ido produciendo a medida que avanzaba la pandemia, las cuidadoras siguen 
verbalizando que es uno de los aspectos que mayor inseguridad les provoca (C6).

\section{Tema: impacto de las medidas preventivas}

La necesidad de instaurar medidas preventivas, tanto a nivel social como en las dinámicas de los centros, ha supuesto un cambio muy restrictivo en las actividades de la vida diaria de estas personas. La adaptación se ha percibido como la necesidad de incorporar nuevas rutinas y la reducción de la interacción social.

\section{Subtema: limitación de la libertad}

Las participantes explican cómo las medidas de confinamiento impuestas han supuesto un gran deterioro de sus relaciones sociales. La palabra libertad está asociada al hecho de poder disponer de tiempo de descanso entre tratamientos. Existe un elemento común que es la relación entre la pérdida de ciertas rutinas, como podía ser controlar los horarios de los transportes o poder disponer como tiempo libre las horas durante las cuales la persona se estaba dializando, con su sensación de limitación personal (C1-C9). La ausencia de centros donde poder realizar las vacaciones es percibida como una gran pérdida (C1). Se han visto obligadas a hacer cambios en la organización y gestión de sus necesidades agravados por la reducción de los apoyos sociales y familiares (C5).

\section{Subtema: aislamiento social y convivencia}

Las cuidadoras no se mostraron especialmente demandantes de cuidados sino de cobertura de ciertas necesidades que antes cubrían con sus propios entornos familiares y sociales $(\mathrm{C} 5, \mathrm{C} 6)$. En relación a los profesionales se mostraban agradecidos por su trabajo y dedicación y especialmente a aquellos profesionales que mantuvieron el contacto mediantes llamadas telefónicas $(\mathrm{Cl}, \mathrm{C} 7, \mathrm{C} 8)$. Muchas cuidadoras han vivido peor el primer confinamiento. El miedo a contagiarse y contagiar a sus familiares hizo que sacrificaran muchos contactos sociales pero han ido estableciendo nuevas dinámicas a medida que esta situación se alargaba en el tiempo (C2, C3, C4, C9). Las cuidadoras manifiestan que tras un periodo inicial de desconcierto volvieron a asumir responsabilidades y se sentían capaces de controlar la situación, esta sensación de control ha sido fundamental para mejorar su situación emocional (C5).

\section{Tema: mortalidad}

De forma casi unánime las cuidadoras han mostrado su conciencia de percepción de que en cualquier momento podían enfermar sus familiares y que dado su estado, eso suponía un riesgo vital. La muerte ha estado presente en la mayoría de las conversaciones como un elemento que tienen muy presente en la patología renal.

\section{Subtema: percepción de riesgo propio y del familiar de morir}

Las cuidadoras tenían más presente, y así lo verbalizan, que sus familiares están en un alto riesgo de contraer la enfermedad y morir, sin embargo su propia muerte ha salido en menor ocasión en las conversaciones. Perciben como un riesgo mayor enfermar y no poder cuidar a sus familiares que su propia muerte (C5-C7). La muerte se muestra como un hecho ineludible y que acontecerá propiciado por la propia enfermedad renal $(C 2, C 3, C 6, C 10)$. Solo una de las entrevistadas hace mención a la fe religiosa como un elemento modulador del riesgo a morir al que se ven sometidos sus familiares $(C 5, C 7)$.

\section{Subtema: muerte de personas próximas}

Hay una vinculación directa entre la verbalización del riesgo a morir y haber vivido fallecimientos próximos ( $\mathrm{Cl}, \mathrm{C} 2, \mathrm{C} 3, \mathrm{C} 4, \mathrm{C} 5, \mathrm{C} 6, \mathrm{C} 7, \mathrm{C} 8, \mathrm{C} 9)$. Ante estas muertes, las cuidadoras reaccionaban con desánimo y resignación, como si en cierta forma el riesgo se fuera asumiendo y se visualizaba que esto podía ocurrir en cualquier momento $\left(\mathrm{Cl}_{1}, \mathrm{C} 5\right)$. Se hace especial mención a personas con las que sus familiares compartían el transporte y lo relacionan con sus quejas hacía un sistema de traslado al que consideran el mayor riesgo para sus familiares $(\mathrm{Cl}, \mathrm{C} 6, \mathrm{C})$.

\section{Tema: enfermedad por COVID-19 y vacunación}

La pandemia ha pasado por diferentes fases y al realizar estas entrevistas un porcentaje muy alto de la población está vacunada con la pauta completa, especialmente la población con patología crónicas y sus familiares. Se ha pedido a las entrevistadas que reflexionaran sobre el impacto que había tenido esta vacunación en su experiencia sobre la enfermedad.

\section{Subtema: riesgo de transmisión}

La mayoría de las participantes han integrado rutinas preventivas en sus actividades de la vida diaria y destacan entre ellas el aumento de la higiene y la utilización de la mascarilla (C1, C2, C4, C7, C9). La limpieza con lejía y el lavado de la ropa de sus familiares cuando vuelven de la diálisis son elementos comunes que aportan sensación de control sobre la transmisión (C2-C3). El elemento que unánimemente ha sido considerado como mejor barrera de protección ha sido la mascari- 
Ila. Todas y cada una de las participantes expresó que utilizarla les daba sensación de seguridad (C1-Cl0). Se expresa cierto conflicto sobre cómo se gestionó al inicio, en los centros, las medidas preventivas, pero esa percepción se diluyó y se han sentido seguras de cómo se gestionaban las medias por parte de las la instituciones y como les habían explicado qué medidas tomar $(\mathrm{C} 2, \mathrm{C} 3, \mathrm{C} 4)$.

\section{Subtema: desconocimiento}

Las cuidadoras han pasado de una primera fase de total desconocimiento sobre la enfermedad a disponer de ciertas nociones de prevención, lo cual les ha permitido tener una percepción de control de la situación. Según su discurso, esa percepción de control ha aumentado al mismo tiempo que se integraban ciertas normas de conducta en la sociedad, así como que su situación personal mejoraba al volver a tener acceso a los soportes familiares. Las cuidadoras y el cuidador viven junto a su familiar, el parentesco es muy próximo siendo mayoritariamente cónyuge. Todas las cuidadoras expresaron su preocupación por el impacto que podía tener la infección en la salud de sus familiares a los que perciben como de gran vulnerabilidad (ClC10). Hay un mayor desconocimiento actualmente sobre los recursos de los que disponen, especialmente frente a situaciones de urgencias de sus familiares (C5-C8). Consideraron que tienen que asumir las medidas impuestas para evitar la propagación pero las consideran muy duras y coartan su libertad, ya de por sí muy limitada por su rol de cuidadoras (C1). Inicialmente se sintieron más desamparadas por el miedo a lo desconocido. La reacción inicial de proteger a los más vulnerables, especialmente gente mayor y con comorbilidades las aisló socialmente, pero han ido recuperando parte de las redes de apoyo mutuo y éstas son también fuente de información sobre la enfermedad $(\mathrm{Cl}, \mathrm{C} 2, \mathrm{C} 3, \mathrm{C} 4, \mathrm{C} 7)$. La posibilidad de contagiar a sus familiares es una preocupación constante, pero la mayoría han ganado en confianza y han recibido apoyo de su entorno cercano.

\section{Subtema: experiencia personal}

Las cuidadoras varían durante la conversación entre el miedo, la impotencia frente a una enfermedad, el orgullo de haberse adaptado y la sensación de haber adquirido un cierto control sobre la situación (C1-C10). Esta experiencia ha sido muy limitante porque ha reducido mucho más sus actividades lúdicas, ya de por sí escasas (C1). Si bien lo han vivido con mucho miedo, no expresan sentir que hayan proporcionado a sus familiares peores cuidados o que los hayan desatendido, sino al contrario, muestran satisfacción de sus estado de salud como una comprobación de que han realizado correctamente sus cuidados $(\mathrm{Cl}, \mathrm{C} 3, \mathrm{C} 4, \mathrm{Cl})$.

\section{Subtema: confianza en la vacuna}

La vacuna es un punto de inflexión en las conversaciones sobre la percepción de riesgo. Hay confianza en la mayoría de las cuidadoras de que ahora es mucho más difícil contraer la enfermedad. Dos de las participantes, sin embargo, muestran su desconfianza relacionada con su efectividad y en cómo se han desarrollado las vacunas (C4-C6).

\section{Subtema: situación post vacunación}

La situación post vacunación se percibe con optimismo moderado $(C 3, C 6)$. Hay un cierto elemento de distensión a la hora de asumir los riesgos, principalmente el traslado a diálisis. Sin embargo, la vacunación no significa, en ningún caso para las cuidadoras, disminuir las nuevas rutinas adquiridas. Se expresa en varias ocasiones que seguirán actuando igual que han actuado hasta ahora (C7-C9).

\section{Tema: emociones y expectativas}

Las emociones están presentes en todas las entrevistas, en diversos grados y en diferentes momentos. Hay un apartado especial que es más emotivo, cuando se describen muertes próximas, de familiares o personas allegadas (C5-C7).

\section{Subtema: grado de miedo y ansiedad}

Hay un miedo latente en la mayoría de las entrevistas, pero que se percibe cómo se va transformando en ansiedad o preocupación a medida que se avanza en la temporalidad de los acontecimientos. Hay una relación directa sobre algunos aspectos claves de las entrevistas, como es la ansiedad que generan los traslados o la seguridad que proporcionan las rutinas (C1-C10).

\section{Subtema: Aumento de hábitos no saludables}

No ha habido cambios muy sustanciales en la mayoría de los casos, pero sí que se hace mención a un mayor grado de ansiedad acompañado de dificultades para dormir (C5-C7). Una de las entrevistadas refiere haber adquirido el hábito de fumar (C1) o cambios en la ingesta que considera como poco saludables (C5).

\section{Subtema: alteración del sueño}

La alteración del patrón del sueño se da en la mayoría de las entrevistadas pero no es percibido como un problema. Una de las cuidadoras menciona que toma 
pastillas para dormir pero que las ha tomado siempre (C5), en otras ocasiones se mencionan remedios naturales.

\section{Subtema: proyecciones futuras}

Destaca en todas las entrevistadas la resiliencia que muestran ante esta situación en sus vidas. Proyectan su futuro teniendo como eje la evolución propia de la enfermedad renal más que la pandemia en sí. Ven el futuro como una cronicidad de esta situación, con estos patrones preventivos ya integrados en sus rutinas, pero solicitan ciertos elementos de apoyo necesarios para cubrir sus necesidades, como son mejoras en el transporte, ayudas sociales y recuperación de sus relaciones sociales.

\section{Discusión}

Cinco argumentos surgieron en una cohorte de cuidadoras primarias de pacientes dependientes en tratamiento de HD sobre el impacto de la pandemia de COVID-19: servicios de salud, medidas preventivas, morbimortalidad de la enfermedad, vacunación y emociones percibidas.

España cuenta con un sistema sanitario público en el que destaca la detección de la enfermedad renal en fases tempranas, la universalidad del tratamiento, que incluye a personas dependientes por edad o por otras causas, y además, al ser uno de los países con tasas de donación y trasplante más altas del mundo, los usuarios contábamos que una atención a la insuficiencia renal de calidad estaba garantizada. Así los cuidadores de pacientes en HD se sentían razonablemente seguros y apoyados por los agentes sanitarios, por lo que no es de extrañar que la pandemia haya trastocado estas expectativas y haya puesto a prueba la resiliencia de un colectivo especialmente vulnerable ${ }^{15}$.

En los momentos iniciales de la pandemia los servicios de nefrología sufrieron la situación general de desconcierto en la que se vio inmerso el mundo sanitario y tuvieron que improvisar protocolos que garantizaran el acceso al tratamiento habitual de los pacientes, la implementación de medidas preventivas en los centros y la educación sanitaria del paciente y su familia para que se adaptaran a la nueva situación ${ }^{4,5,16}$.

Los pacientes en tratamiento sustitutivo y sus cuidadores se han visto sometidos a la misma presión y restricciones que la población general, en cambio, sus vivencias han sido condicionadas por las características de una situación previa especialmente delicada. Las informantes de este estudio refieren que el miedo y desconcierto se hicieron patentes en las primeras fases de la pandemia y destacan especialmente el temor a que su familiar no pudiera acudir a recibir tratamiento en su centro habitual y con la misma frecuencia que lo hacía anteriormente. Además, refieren cierto miedo ante la posible dificultad para acceder a otros servicios sanitarios si su familiar lo necesitase? Se ha de tener en cuenta que hubo que reorganizar la ubicación de muchos pacientes para rentabilizar los recursos y priorizar la atención a pacientes graves $^{7}$. También los pacientes renales son demandantes frecuentes de otros servicios que estaban colapsados y faltos de personal o de materiales, de aquí que la preocupación inicial se centrara en recibir la asistencia necesaria. Por otra parte, el personal sanitario ha redistribuido competencias para ser autosuficientes y no sobrecargar otros servicios ${ }^{17}$.

El transporte colectivo establecido para acudir a recibir el tratamiento es referido como un problema añadido, ya que las entrevistadas son conscientes de que aumenta la posibilidad de contagio al trasladar a varios pacientes, además de variar rutas, horarios y el tiempo de desplazamientos, de aquí la decisión de algunos de optar por un vehículo privado, en algunos de los casos con grandes dificultades debido a la situación de dependencia del familiar. Los problemas derivados del traslado a los centros son recurrentes en situación de "normalidad" y la pérdida de tiempo y costes son objeto de quejas y reclamaciones, por tanto en situaciones críticas adquiere una mayor dimensión ${ }^{6,18-21}$.

Se considera rápida y satisfactoria la atención recibida por parte de los centros de diálisis, que pese al desconcierto inicial, la falta de medios y de personal, establecieron normativas claras sobre la actuación dentro del propio centro, haciéndolo extensivo a los cuidados en el hogarb. Estas iniciativas les hicieron sentirse más protegidos e informados que la población general y ha ayudado a adaptarse a la nueva situación, por lo que manifiestan agradecimiento por la ayuda recibida. La cercanía y cuidados que se dan en el entorno de la atención al paciente renal facilita una relación personal muy frecuente. El conocimiento que tenemos los profesionales sanitarios de las características de pacientes y familia ha sido sin duda determinante para establecer una buena comunicación y un mejor cumplimiento de las medidas preventivas ${ }^{20}$. 
Se acusa en las entrevistas la falta de otro tipo de ayudas sociales y de información, que se han visto desbordadas por la situación y, por tanto, el centro de diálisis y sus servicios han sido punto de referencia y de apoyo para los pacientes y sus allegados.

El miedo al contagio y a la muerte continúan presentes en este colectivo, que por otra parte es conocedor de su fragilidad y limitada esperanza de vida, son conocedores de que la mortalidad ha sido muy significativa tanto entre su grupo etario como en pacientes renales ${ }^{22}$. Como está descrito entre los cuidadores de personas con patología crónicas, éstas perciben como un gran riesgo el caer ellas enfermas y no poder hacerse cargo de su familiar, lo cual les lleva a ser estrictas con las medidas de prevención que procuran no descuidar y que de igual forma, el personal de los centros refuerza regularmente ${ }^{23}$.

Se refiere el cambio de hábitos y la pérdida de libertad como una consecuencia natural que les afecta poco, ya que este colectivo tiene una vida social que ya estaba muy limitada por la edad, los cuidados y la administración del tratamiento ${ }^{12}$. Son conscientes del mayor riesgo de contagio y refieren mayores dificultades para relacionarse con familias y amigos, pero el permanecer juntos cuidadores y pacientes no ha llevado a situaciones dramáticas de aislamiento, como ha ocurrido con otros colectivos frágiles como las personas institucionalizadas ${ }^{24}$. Las cuidadoras acusan la pérdida de los pocos momentos de ocio que tenían antes de la pandemia y la dificultad para trasladarse, especialmente para ir de vacaciones, desde siempre complicada y engorrosa, pero en el momento de las entrevistas casi imposible.

Respecto al futuro, tienen unas expectativas limitadas por su situación al margen de la pandemia, confían relativamente en la vacuna que, aunque se administró precozmente, es sabido que con los pacientes renales tiene una eficacia limitada y menor que en la población general ${ }^{25}$, de aquí la importancia de continuar con las medidas de prevención.

En general manifiestan su satisfacción por haber sido capaces de afrontar la situación con entereza, y atribuyen parte de este mérito a su experiencia previa como cuidadoras y a la formación recibida.

\section{Fortalezas}

La mayoría de los estudios cualitativos existentes sobre el COVID-19 son estudios sobre los propios enfermos renales y como les ha afectado la enfermedad. Por el contrario, nuestro estudio se centra en las cuidadoras primarias y abarca desde el periodo inicial de máxima incidencia hasta el periodo post vacunación. El estudio se apoya en investigaciones anteriores que mostraban el agotamiento del cuidador principal y cómo era capaz de crear rutinas para adaptarse a la enfermedad, en la presente investigación se pide que expliquen sus vivencias con total libertad y que describan cómo han evolucionado sus emociones durante todo este tiempo. Divergiendo de los resultados de muchos estudios sobre niveles de estrés y agotamiento emocional, descubrimos que estas personas tienen una gran capacidad de adaptación y resiliencia incluso en situaciones de excepcionalidad.

\section{Limitaciones}

Debido a las características de la investigación cualitativa, el tamaño de la muestra de este estudio fue limitado. En primer lugar, la mayoría de los participantes eran cuidadoras, incluyendo solo a un hombre. Las experiencias ligadas al género, deben explorarse más a fondo. En segundo lugar, debido a la naturaleza de la prevención no pudimos realizar entrevistas personales perdiéndose matices importantes para la investigación cualitativa. Además, este estudio fue un estudio a corto plazo, en un periodo muy concreto y pidiendo que resumieran la experiencia de dos años con muchas vivencias. La experiencia a largo plazo de los sujetos de investigación sería una vía valiosa para explorar en el futuro.

\section{Conclusiones}

Este estudio proporcionó una comprensión de las experiencias de vida de las cuidadoras primarias de pacientes en HD durante la pandemia por COVID-19 a través de un enfoque fenomenológico. En la etapa álgida del brote epidémico las vivencias de las cuidadoras fueron provocadas por el desconocimiento, por no saber actuar adecuadamente, y el miedo al contagio. Como positivo afirman sentirse apoyadas y adaptadas a la situación adoptando medidas de prevención adecuadas para mantener a salvo a su familiar. Aprecian la seguridad proporcionada por el personal de los centros de diálisis, que asumieron competencias de educación sanitaria y apoyo emocional, convirtiéndose en referentes y les preocupa las dificultades inherentes a los traslados y la falta de ayuda de otras estructuras sociales. Son conscientes de su propia fragilidad y la de la persona cuidada y no perciben grandes cambios en su forma de vida ni en su esperanza de futuro, pese a la vacunación y otros adelantos científicos. Es necesario profundizar en las necesidades y demandas específicas de las cuidadoras para asegurar el soporte necesario a este colectivo de pacientes especialmente vulnerables. 
Agradecimientos: A las cuidadoras participantes y a la Sociedad Española de Enfermería Nefrológica (SEDEN).

Recepción: 12-08-21

Aceptación: 30-08-21

Publicación: 30-09-21

\section{Bibliografía}

1. Zhu N, Zhang D, Wang W, Li X, Yang B, Song J, et al. A novel coronavirus from patients with pneumonia in China, 2019. N Engl J Med. 2020;382(8):727-33.

2. Khalid I, Khalid TJ, Qabajah MR, Barnard AG, Qushmaq IA. Healthcare workers emotions, perceived stressors and coping strategies during a MERSCoV outbreak. Clin Med Res. 2016;14(1):7-14.

3. Condes, Emilia; Arribas JR. Impact of COVID-19 on Madrid hospital system Impacto. Enferm Infecc Microbiol Clin. 2021;39:256-64.

4. Maldonado $M$, Ossorio $M$, Del Peso G, Santos $C$, Álvarez L, Sánchez-Villanueva $R$, et al. Incidencia y resultados de la COVID-19 en una unidad de diálisis domiciliaria en Madrid (España) durante el pico de la pandemia. Nefrologia. 2021;41(3):329-36.

5. Albalate $M$, Arribas $P$, Torres $E$, Cintra $M$, Alcázar $\mathrm{R}$, Puerta $\mathrm{M}$, et al. Alta prevalencia de COVID-19 asintomático en hemodiálisis. Aprendiendo día a día el primer mes de pandemia de COVID-19. Nefrología. 2020;40(3):279-86.

6. Arenas MD, Villar J, González C, Cao H, Collado S, Crespo $\mathrm{M}$, et al. Manejo de la epidemia por coronavirus SARS-CoV-2 (COVID-19) en unidades de hemodiálisis. Nefrología. 2020;40(3):258-64.

7. GOBIERNO DE ESPAÑA-MINISTERIO DE SANIDAD. Documento técnico Recomendaciones para el manejo, prevención y control de COVID-19 en Unidades de Diálisis. Sociedad Española de Nefrología. 2020.
8. Candel FJ, San-Román J, Barreiro P, Canora J, Zapatero $A$, Carretero $M$, et al. Integral management of COVID-19 in Madrid: Turning things around during the second wave-Authors' reply. Lancet Reg Heal - Eur. 2021;3:100076.

9. Alberici F, Delbarba E, Manenti C, Econimo L, Valerio $F$, Pola $A$, et al. Management of Patients on Dialysis and With Kidney Transplantation During the SARS-CoV-2 (COVID-19) Pandemic in Brescia, Italy. Kidney Int Reports. 2020;5(5):580-5.

10. Sun $N$, Wei $L$, Shi S, Jiao D, Song $R, M a ~ L$, et al. A qualitative study on the psychological experience of caregivers of COVID-19 patients. Am J Infect Control. 2020;48(6):592-8.

11. Losada A, Vara-García C, Romero-Moreno R, Barrera-Caballero $S$, Pedroso-Chaparro $M$ del $\mathrm{S}$, Jiménez-Gonzalo $\mathrm{L}$, et al. Caring for Relatives with Dementia in Times of COVID-19: Impact on Caregivers and Care-recipients. Clin Gerontol. 2021.

12. Carmona Moriel CE, Nolasco Monterroso C, Navas Santos L, Caballero Romero J, Morales Medina P. Análisis de la sobrecarga del cuidador del paciente en diálisis peritoneal. Enferm Nefrol. 2015;18(3):180-8.

13. Sánchez-Álvarez JE, Pérez-Fontán $M$, Jiménez-Martín C, Blasco-Pelícano M, Cabezas-Reina CJ, Sevillano-Prieto AM, et al. Situación de la infección por SARS-CoV-2 en pacientes en tratamiento renal sustitutivo. Informe del Registro COVID-19 de la Sociedad Española de Nefrología (SEN). Nefrologia. 2020;40(3):272-8.

14. Magaly D, Cadenas R. El Rigor en la Investigación Cualitativa: Técnicas de Análisis, Credibilidad, Transferibilidad y Confirmabilidad. Sinop Educ Rev Venez Investig. 2016;7(1):17-26.

15. Martínez DH, Zambrano DÁ, Silva EA, Moreno SC. Needs of people in dialysis therapy and their caregivers: Integrative review. Enferm Nefrol. 2018;21(2):155-65.

16. Arribas-Cobo $P$, Bernabé-Villena $M$, Martínez-Dios I, Ruiz-Almería S, Díaz de Argote-Cervera P. Estudio descriptivo del primer mes de situación de pandemia por COVID-19 en una unidad 
de diálisis hospitalaria. Enfermería Nefrológica. 2020;23(2):133-47.

17. Andreu-Periz D, Ochando-García A, Limón-Cáceres E. Experiencias de vida y soporte percibido por las enfermeras de las unidades de hemodiálisis hospitalaria durante la pandemia de COVID- 19 en España. Enfermería Nefrológica. 2020;23(2):14859.

18. Traver J, Barril G. ¿Se puede mejorar el problema del transporte en hemodiálisis? Nefrologia. 1998;XVIII(2):176-7.

19. Capillas Echevarria B, De Baldeón Herrero MSD, Arrausi Larrea A, Etura Ule E, González Manjon M, Echavarri Escribano M. Traslado en ambulancia, alargamiento de tiempo inevitable en cada sesión de diálisis. Rev la Soc Esp Enferm Nefrol. 2007;10(3):248-9.

20. Gómez-Beltrán PA, Domínguez Carranza E, Medero Rubio F, Pérez Márquez M. Recomendaciones para el manejo del paciente con enfermedad renal crónica ante el SARS-CoV-2. Enfermería Clínica. 2021 Feb;31(supl 1):S55-61

21. Soler MJ, Macia Heras M, Ortiz A, del Pino y Pino MD, Salgueira Lazo M. Impacto de la pandemia COVID-19 en los servicios de Nefrología españoles. Nefrología. 2020;40(6):579-84.
22. Carrillo-Algarra AJ, Duque Salazar N, Nieto Parra KJ. La evidencia cualitativa y el cuidado del paciente en diálisis peritoneal. Enfermería Nefrológica. 2016;19(2):154-63.

23. Alexopoulos $P$, Soldatos $R$, Kontogianni E, Frouda $M$, Loanna Aligianni S, Skondra $M$, et al. COVID-19 Crisis Effects on Caregiver Distress in Neurocognitive Disorder. J Alzheimer's Dis. 2021;79(1):459-66.

24. Pinazo-hernandis S. Impacto psicosocial de la COVID-19 en las personas mayores: problemas y retos. Rev Esp Geriatr Gerontol. 2020;55(5):249-52.

25. Martín de Francisco AL. Resultados de la vacunación COVID-19 en pacientes en diálisis y trasplantados de riñón. Nefrología al día [Internet] May 2021 [consultado 05 sept 2021]; [aprox. 8 p]. Disponible en: https://static.elsevier.es/nefro/ monografias/1/397/397_170520212210.pdf.

Este artículo se distribuye bajo una Licencia Creative Commons Atribución-NoComercial 4.0 Internacional. https://creativecommons.org/licenses/by-nc/4.0/ 